\title{
Researching to Determine the Characteristic Parameters of the Power Ultrasonic Transducer by Finite Element Method and COMSOL-MULTIPHYSICS Program
}

\author{
Nguyen Van Thinh \\ The University of Technology and Education \\ The University of Danang \\ Danang, Vietnam
}

\author{
Hoang Thi My Le \\ The University of Technology and Education \\ The University of Danang \\ Danang, Vietnam
}

\begin{abstract}
The power ultrasonic transdurce operates in range of frequencies from $18 \mathrm{KHz}$ to $45 \mathrm{kHz}$ [9]. Depending on the material, the tranducer structure has the characteristic parameters that suitables for each application. The research results in this paper: building the power ultrasound transducer model from the PZT hard piezoelectric materials and using finite element method (FEM - Finite Element Method) and COMSOL- Multiphysics (CM) prgram determine characteristic parameters. The research result has identified resonant oscillation frequency, the transducer displacement and the characteristics of the sound pressure emanating from the ultrasound transducer. These results are fundamental for the designing and applying in the high power ultrasound techniques..
\end{abstract}

Keywords-Langevin tránducer; PZT hard piezoelectric ceramic ; sound pressure field; FEM; COMSOL-Multiphysics

\section{INTRODUCTION}

The Langevin ultrasound transducer is a key element using to make high power ultrasonic devices. Depending on the material and the design model, the ultrasonic transducer has different characteristic parameters. In order to achieve the specific application purpose, it is necessary to determine the ultrasonic transducer characteristic parameters. By constructing PZT doped hard piezoelectric ceramic models and Langevin transducer, using finite element method in the PZT hard piezoelectric material environment, COMSOLMultiphysics program survey the dependence of characteristic parameters according to frequency. In this papper, we simulated to determinate the oscillation characteristic, the resonance frequency of the PZT hard piezoelectric ceramic, the Langevin transducer displacement and the sound pressure field to emanate rom the front of the Langevin transducer. Research results are the basis for selecting materials, designing plans and applications.

\section{FINITE ELEMENT METHOD IN PIEZOELECTRIC ENVIRONMENT [4], [6], [9]}

Finite element method is applied to analyse the characteristic piezoelectric transducer parameters with different design models. In order to achieve accurate analytical results, it is necessary to set up mathematical and physical equations and impose boundary condition on each case.

\section{A. Continuous equations in piezoelectric environment}

$$
\begin{aligned}
& \{T\}=\left[c^{E}\right]\{S\}-[e]\{E\} \\
& \{D\}=[e]+\{S\}+\left[\varepsilon^{S}\right]\{E\}
\end{aligned}
$$

In which, $\{T\},\{D\},\{E\}$ are the stress vector, the electric displacement vector and the electric field intensity vector respectively. $[e],\left[\varepsilon^{S}\right],\left[c^{E}\right]$ are the piezoelectric coefficient matrix, the dielectric constant matrix and the stiffness matrix respectively. Index $\uparrow$ symbolizes for matrix or shift vector Index " $\dagger$ " symbolizes for matrix or shift vector. The mechanical terms in equation (1) follow 2 Newton's law, according to (3).

$$
\frac{\rho \partial^{2}\{\mathbb{R}\}}{\partial t^{t}}=-\{T\}
$$

With $\mathbb{R}=\{u v w\}^{\dagger}$ is mechanical displacement vector along axes $x, y$ and $z$. The $\{\mathrm{S}\}$ strain vector relates the $\{\mathrm{R}\}$ the displacement vector according to the expression according to the expression:

In which:

$$
\{S\}=\left[\mathrm{B}_{\mathbf{R}}\right]\{\mathbb{R}\}
$$

$$
\left[\mathrm{B}_{\mathbf{R}}\right]=\left[\begin{array}{cccccc}
\frac{\partial}{\partial x} & 0 & 0 & 0 & \frac{\partial}{\partial z} & \frac{\partial}{\partial y} \\
0 & \frac{\partial}{\partial y} & 0 & \frac{\partial}{\partial z} & 0 & \frac{\partial}{\partial x} \\
0 & 0 & \frac{\partial}{\partial z} & \frac{\partial}{\partial y} & \frac{\partial}{\partial x} & 0
\end{array}\right]
$$

The electric terms in (1) follow Gauss's law, with the assumption that piezoelectric materials are insulating materials, no charge current running in the transducer.

$$
\nabla .\{D\}=0
$$

The $E$ electric field is related to the $\Theta$ voltage by

$$
\{E\}=-\{B\} \Theta
$$

with, $\quad\left\{B_{\Theta}\right\}=\left\{\frac{\partial}{\partial x} \frac{\partial}{\partial y} \frac{\partial}{\partial z}\right\} \dagger$

In which, $\left\{\sigma_{G}\right\}=\{T D\} \dagger,\left\{\varepsilon_{G}\right\}=\{\mathrm{S}-\mathrm{E}\} \dagger$ are the stress vector and the general deformation vector respectively. The [c] general elastic matrix form: 


$$
[c]=\left[\begin{array}{cc}
c^{E} & e \\
e^{T} & -\varepsilon^{S}
\end{array}\right]
$$

The stress and strain relationships are represented by the equation:

$$
\left\{\varepsilon_{G}\right\}=\left[B_{G}\right]\left\{\mathbb{R}_{G}\right\}
$$

In which, $\quad\left\{\mathbb{R}_{G}\right\}=\{\mathbb{R} \Theta\} \dagger=\left\{\begin{array}{llll}u & v & \boldsymbol{1} & \boldsymbol{1}\end{array}\right\}^{\dagger}$ is the general displacement vector and the general matrix with form:

$$
\left[B_{G}\right]=\left[\begin{array}{cc}
B_{\mathbb{R}} & 0 \\
0 & B_{\Theta}
\end{array}\right]
$$

\section{B. Dynamic equations in piezoelectric environment}

With piezoelectric materials that have satisfied the continuous equations, the $\mathbb{R}_{G}$ general displacement of any approximated point by $[\mathbb{N}]$ displacement function and the $\{\mathfrak{R}\}$ vector containing the finite displacement nodes.

$$
\left\{\mathbb{R}_{G}\right\}=[\mathbb{N}]\{\mathfrak{R}\}
$$

Đối với bài toán này, tại nút thứ $\mathrm{h}$ có 4 bậc tự do. Trong hệ tọa độ Descartes 3 chiều vector của nút thứ h có dạng.

For this problem, at the hode has the 4 freedom degrees. In the 3-dimensional Descartes coordinate system, the $\left\{\Re_{h}\right\}$ vector of the $\mathrm{h}^{\text {th }}$ node is the form:

$$
\left\{\mathfrak{R}_{h}\right\}=\left\{\mathbb{R}_{h} \Theta_{h}\right\} \dagger=\left\{u_{h} v_{h} w_{h}\right\}^{\dagger}
$$

Mathematical, physical basis for determining piezoelectric material parameters are described by general equations (1) $\div(13)$.

An arbitrary problem is solved by imposing boundary conditions, algorithm of finite element analysis based on input data as matrix of material coefficients: elasticity coefficient, piezoelectric coefficient and dielectric constant. The resonant oscillation frequency and characteristics are obtained by combining individual solutions.

C. Building the Langevin transducer model [2], [3], [5], [8]

Figure 1, describing the Langevin transducer structure consists of two PZT hard piezoelectric ceramic plates. The front is an aluminum metal block. The back is a metal block of steel. The whole transducer is linked by steel bolt.

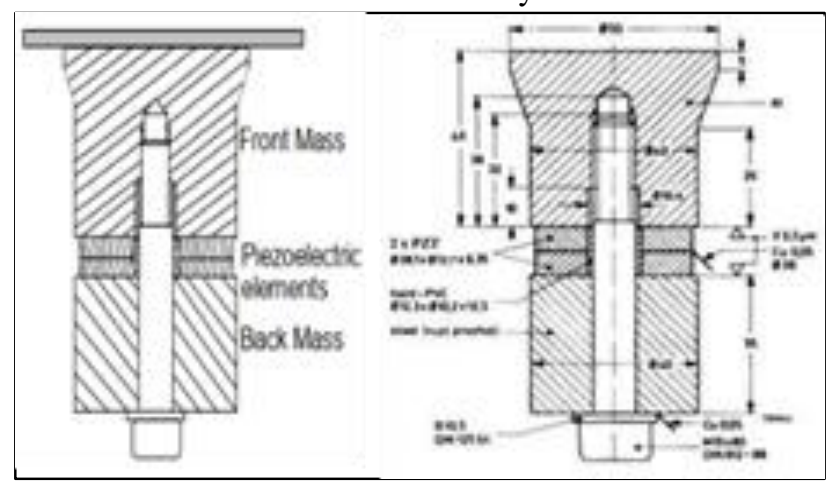

Fig. 1. The Langevin transducer structure [3]

\section{SURVEYING THE LANGEVIN TRANSDUCER CHARACTERISTIC PARAMETERS BY COMSOL - MUTIPHYSICS PROGRAM}

A. Setting the piezoelectric ceramic plate model and sound pressure field

Figure 2 is the oscillation survey model according to the different frequencies of the PZT piezoelectric hard ceramic to be built from the COMSOL - Multiphysics program, the meshes and nodes distribution with FEM. Sizes: the $40 \mathrm{~mm}$ outer diameter, the $16 \mathrm{~mm}$ internal diameter and the $5 \mathrm{~mm}$ thickness that are also the actual sizes to manufacture the Langevin transducer.

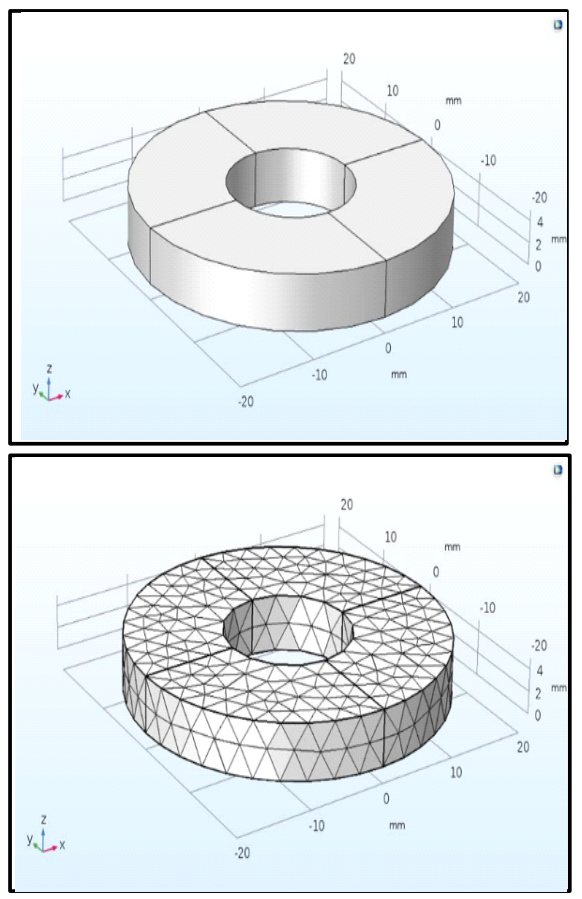

Fig. 2. The PZT piezoelectric ceramic plate model distributing the meshes and buttons with FEM

The model surveys the displacement and the emitted sound pressure field from the front of the Langevin transducer according to different frequencies, in Figure 3.

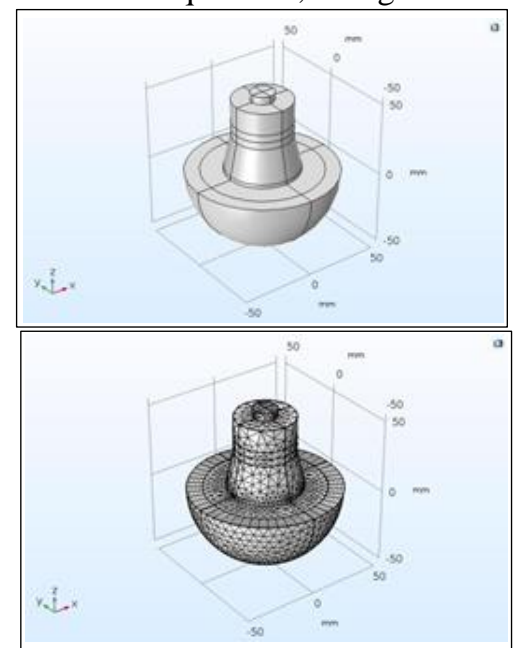

Fig. 3. The surveying model of the displacement and the sound pressure field

After the built model, we simulate the hard PZT piezoelectric ceramic operation and the Langevin transducer 
according to frequency. The simulation process purpose is to determine the resonant frequency, the displacement dependence according to the resonant frequency, the emitted sound pressure field characteristic from the Langevin transducer front.

\section{B. The simulation results to determine the PZT piezoelectric} ceramic plate resonant frequency [7], [2]

Determining the resonance frequency, we surveyed the PZT hard piezoelectric ceramic plate oscillation state in the frequency range from $16.855 \mathrm{kHz}$ to $44.334 \mathrm{kHz}$.

Figure 4 shows the resonant oscillation dependence of the PZT piezoelectric ceramic plate by frequency. The simulation result at the $40 \mathrm{kHz}$ oscillation frequency of the ceramic plate according to the radius in the Figure 4.c is the best match. The ceramic plate oscillates according to the thickness from $16.855 \mathrm{kHz}$ to $27.5 \mathrm{kHz}$ frequencies (Figure 4.a, b) and to be twisted at the $44.334 \mathrm{kHz}$ frequency.

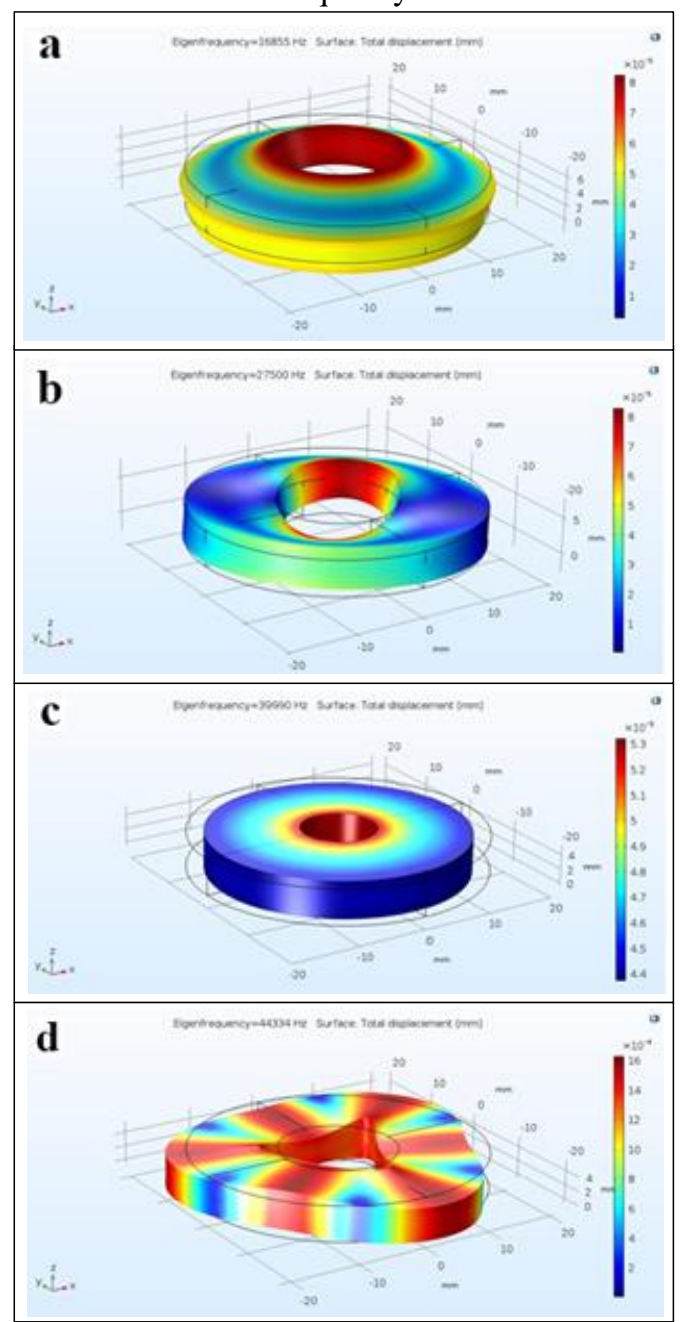

Fig. 4. The PZT piezoelectric ceramic plate resonance oscillation dependence according to frequency

The oscillation spectrum represents the PZT hard piezoelectric impedancedependence by frequency in the Figure 5. The result shows that the impedance reaches the minimum value (Zmin) at the $40 \mathrm{kHz}$ frequency and the resonance phenomenon starts happening. This result is completely suitable for the PZT piezoelectric ceramic plate resonance oscillation at $40 \mathrm{kHz}$ frequency (Figure 4.c).

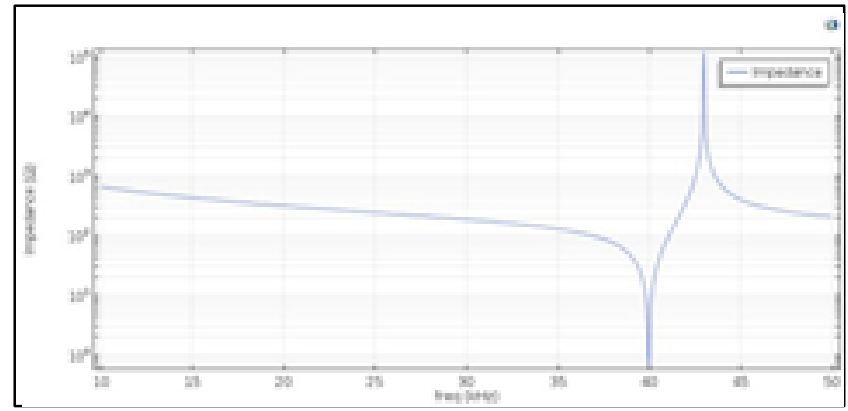

Fig. 5. The PZT piezoelectric ceramic plate impedance dependence by frequency

C. The simulation result to determine the displacement of the Langevin transducer [2], [1]

The results in Figure 6.c and Figure 7 show that the transducer displacement is maximum at $40 \mathrm{kHz}$ frequency.

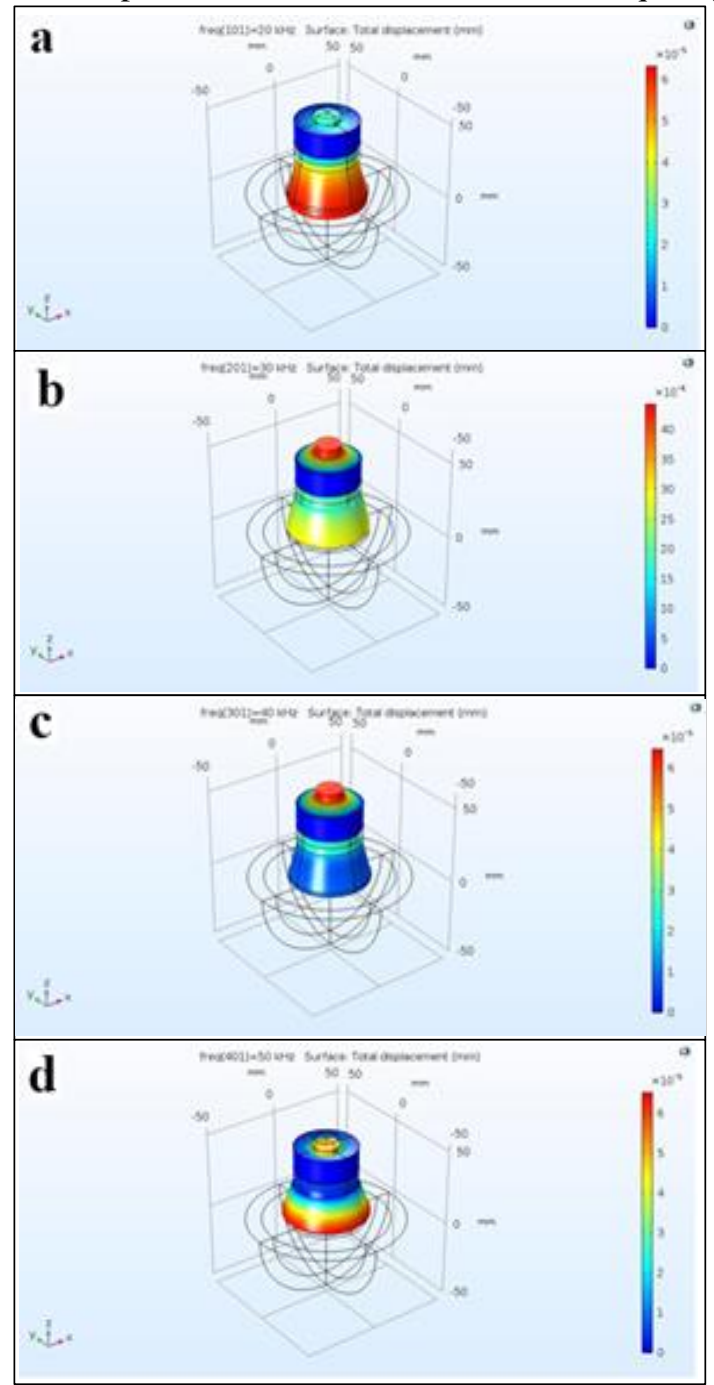

Fig. 6. The transducer displacement dependence by frequency 


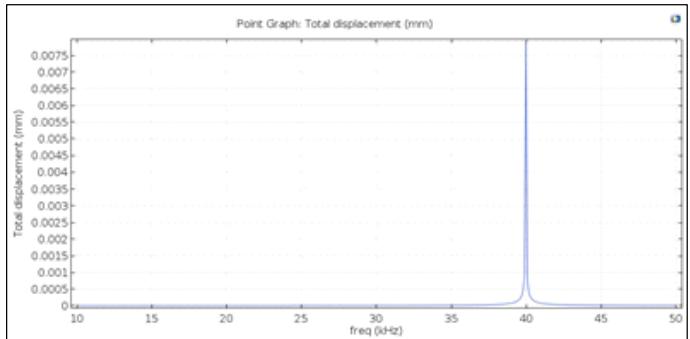

Fig. 7. The oscillation spectrum represents the transducer displacement by frequency

D. The survey result to determine the sound pressure field of the Langevin transducer front [1], [2]

Figure 8 is the simulation result of the emitted sound pressure field from the Langevin transducer front. The sound pressure field clearly shows the compression - expansion process, the spread of the ultrasonic wave to the frequency dependence environment. At the $40 \mathrm{kHz}$ frequency, the sound pressure is maximun at the center and widened the boundary with the best orientation, Figure $8 \mathrm{c}$.

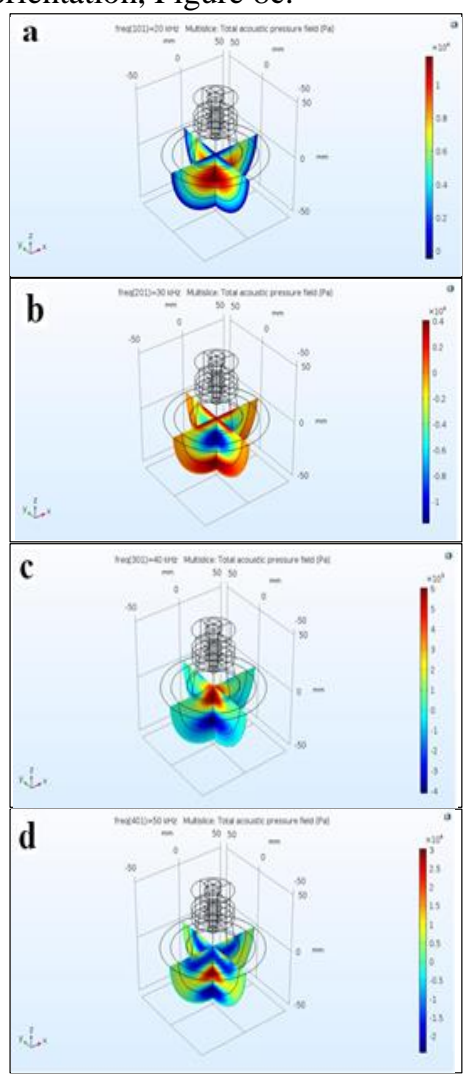

Fig. 8. The sound pressure field of the Langevin transducer front

\section{CONCLUSION}

By finite element method, we have established the continuous equations, kinetic equations, the elastic coefficient matrix , piezoelectric coefficient, dielectric constants of piezoelectric environment.

Using the COMSOL-Multiphysics program builds the model to survey the characteristic parameters of the PZT hard piezoelectric ceramic plate and the Langevin ultrasonic transducer.

The resonant frequency of the PZT hardened piezoelectric ceramic is determined at $40 \mathrm{kHz}$. At this frequency the displacement and the sound pressure field are maximum and the best orientability.

\section{REFERENCES}

[1] C. H. Sherman, J. L. Butler, Transducers and Arrays for Underwater Sound.: Springer Science+Business Media, LLC, 2016.

[2] COMSOL Inc. (2011), Introduction to Comsol Multiphysics.: 19982017 COMSOL

[3] Dong Chen, Sanjay K. Sharma, Ackmez Mudhoo (2012), "Handbook on Applications of Ultrasound", Taylor \& Francis Group, LLC

[4] H. Allik and T. J. R. Hughes (1970), "Finite element method for piezoelectric vibration," International Journal of Numerical Methods in Engineering, vol. 2, pp. 151-1 57.

[5] Juan A. Gallego-Juarez and Karl F. Graff (2015), "Power Ultrasonics Applications of High-intensity Ultrasound, Woodhead Publishing.

[6] M. Naillon, R. H. Coursant and F. Besnier (1983), "Analysis of piezoelectric structures by a finite element method," Acta Electronica, vol. 25 , pp. $341-362$.

[7] N. Guo and P. Cawley (1992), "The Finite Element Analysis Of The Vibration Characteristics Of Piezoelectric Discs," Journal of Sound and Vibration, vol. 159, no. 1, pp. 115-1 38.

[8] R. S. Woollett, "Theory of the piezoelectric flexural disk transducer with applications to underwater sound," USL research report No.490 SF-001 0304-1, U. S. Navy Underwater Sound Laboratory 1960.

[9] Y. Kagawa and T. Yamabuchi (1976), "A finite element approach to electromechanical problems with an application to energy-trapped and surface-wave devices," Transactions of Sonics and Ultrasonics $S U-U$, vol. 3, pp. 263-272. 